\title{
EL CONCEPTO DE EXTENSIÓN UNIVERSITARIA EN LA ERA DE LA INFORMACIÓN EL CONOCIMIENTO Y DE CRISIS MULTIFACÉTICAS.
}

David Moreira, Rosa González, Jéssica Zambrano, Carla Félix Silva 


\title{
EL CONCEPTO DE EXTENSIÓN UNIVERSITARIA EN LA ERA DE LA INFORMACIÓN EL CONOCIMIENTO Y DE CRISIS MULTIFACÉTICAS
}

\author{
David Moreira $^{(1)}$, Rosa González $^{(1)}$, Jéssica Zambrano ${ }^{(1)}$, Carla Beatriz Félix Silva ${ }^{(2)}$ \\ Escuela Superior Politécnica Agropecuaria de Manabí. "Manuel Félix López”(ESPAM MFL) ${ }^{(1)}$ \\ Universidad Estatal Península de Santa Elena (UPSE) ${ }^{(2)}$ \\ La Libertad-Ecuador \\ dmoreira@espam.edu.ec
}

\begin{abstract}
Resumen
A pesar de la gran cantidad de definiciones que existen de la Extensión Universitaria aún el concepto no está acabado, lo que imposibilita su comprensión como proceso sustantivo clave para lograr la vinculación de la universidad a la sociedad, también, la conceptualización de los modelos existentes no reflejan la esencia de dicha vinculación. Todo ello ha traído, diferentes insuficiencias, que atentan contra la pertinencia y calidad de la educación superior. En el trabajo se llega a una conceptualización de la extensión universitaria y se da una base para la clasificación de sus modelos..
\end{abstract}

Palabras Claves: Incluya Extensión universitaria, modelos de extensión universitaria, calidad y pertinencia de la educación superior.

\begin{abstract}
Despite the large number of definitions for University Outreach, the concept remains unfinished, which precludes its understanding as a substantial process that is key for universities to reach out to society. Moreover, the conceptualization of the existing models does not reflect the essence of such outreach. As a consequence, different insufficiencies have emerged, which are jeopardizing the purpose and quality of higher education. This study proposes a conceptualization of university outreach, and sets a foundation for model classification.
\end{abstract}

Keywords: University outreach, university outreach models, quality and purpose of higher education.

\section{Introducción}

En la Pedagogía, investigadores que han incursionado en el proceso extensionista han planteado múltiples definiciones sobre este particular, entre los que se encuentran: Álvarez de Zayas (1996), Arce (2005), Barrios (2006), Betto (2012), Bustos (2010), Cedeño (2013),Claret del Valle (2009), Cruz, (1998), Del Huerto (2000, 2007), Doria (2009), Freire (1972), Gazzola (2011), González (1996), GonzálezMoreno (2006), González (2010, 2011, 2012), Hart (1996), Hernández (2011), Horruitiner (2008), Reyes (2007), Sánchez (2003), Serna (2007), Tünnerman (1998), Vilá y et al (2006), Zulueta (2010). Sin embargo pese a la multitud de definiciones aportadas, aun no existe una definición acabada, que recoja las necesidades actuales de desarrollo.
En tal sentido y en estrecho vínculo con lo anterior Betto (2012) explicaba que la extensión universitaria en los tiempos actuales debe seguir varios puntos fundamentales:

- Llevar la universidad a la realidad popular, no para enseñar, no para hacer cosas, sino para involucrase en los procesos del pueblo.

- Ir al pueblo para permitir que el pueblo traiga su enseñanza pero no darlo por un proceso de oralidad sino por un proceso de experiencias, de luchas, de vivencias, las fiestas, la devoción religiosa, el deporte, una dimensión lúdica, gratuidad del universo lúdico.

- Traer el pueblo para dentro de la universidad, traer la universidad para dentro, más bien hacer intención universitaria. La gente con sus experiencias, su cultura.

- Saber de la historia de los sindicatos que están al lado de la universidad, de la historia de los 
antiguos profesores de la universidad, trabajo de memoria de la universidad. (4).

\section{Desarrollo}

En el ámbito de las Ciencias Pedagógicas se ha escrito mucho sobre la extensión universitaria, en tal sentido, abundan innumerables definiciones del término, alguna de las cuales han olvidado la misión de la universidad, sobre todo aquellas, que se identifican con un vínculo unidireccional en la relación universidad-sociedad, también existen algunas, que solamente conciben la dimensión artística y deportiva, otras olvidan el liderazgo que deben ejercer las universidades en la región donde están enclavadas, sin embargo, en la situación actual donde se asienta y se ramifica cada día más el socialismo del siglo XXI, se revelan imperfecciones en el concepto, que están en contradicción con los principios del dicho socialismo.

Se han seleccionado algunas definiciones hechas por reconocidos especialistas de la pedagogía de la educación superior en diferentes momentos históricos, para analizar los elementos que las conforman.

A partir de la segunda mitad del siglo XVIII y principios del XIX, con el incremento de las fuerzas productivas, el aumento de la producción y el mercado, producto del surgimiento del capitalismo, la sociedad requirió, de la presencia de múltiples especialistas que dieran respuesta a las necesidades fundamentalmente industriales, en especial. En ese contexto se introduce la concepción napoleónica de la universidad, cuyo énfasis, positivo en ese momento, está en la formación de profesionales con un buen dominio tecnológico, que dieran respuesta a la situación que el proceso industrial requería. ()Mariás, J.(1957).

Hacia 1790, esta situación requirió de la preparación de profesionales en corto espacio de tiempo, ese fue el momento del nacimiento de la extensión universitaria en Gran Bretaña, según lo ubican diferentes autores ()Sastre, P. Zubiría, A. D’Andrea, R. (2012). no se adoptó una definición, pues se consideró que era una contribución a la universidad a la formación de adultos, que fueron los primeros programas en desarrollarse, extendiéndose posteriormente, a la educación de sectores más amplios de la población, especialmente hacia los trabajadores.()

Al considerarse, como objetivo de la universidad, satisfacer las necesidades de las nacientes industrias, la extensión universitaria tomó un carácter economicista y empresarial. En 1876, se inaugura la Asociación de la Institución Libre de Enseñanza junto con un grupo de catedráticos separados de la Universidad Central de Madrid por defender la libertad de cátedra y negarse a ajustar sus enseñanzas a cualquier dogma oficial en materia religiosa, política o moral, tuvieron que proseguir su labor educativa al margen del Estado creando un establecimiento educativo privado laico (ILE), que impuso el sistema de educación integral, aplicando los métodos intuitivos de Friedrich Fröbel. Incorporaba el trabajo de talleres, la educación física y la "extensión universitaria", con la organización de cursos y conferencias, que se establecería a propuesta de Rafael Altamira y Crevea en 1892. ()Jiménez-Landi, A. (2010).

A partir de estas ideas, se asientan las bases para un futuro distinto, reconociéndose la función social de la universidad, así, en 1898 en la universidad de Oviedo el profesor Leopoldo Alas propone lo siguiente:

"...teniendo en cuenta los trabajos que en todas las partes, fuera de España, se realizan a favor de la cultura popular...la Universidad de Oviedo emprenda desde ahora la obra utilísima llamada extensión universitaria" ()Ferrer, T (1997).

Un año después, Rafael Altamira, en la inauguración del curso de Extensión Universitaria de Oviedo en 1899, se formuló la siguiente definición:

"La Extensión Universitaria comprende: conferencias, que "vulgarizan" los conocimientos científicos, excursiones artísticas y arqueológicas, cursos para obreros en núcleos rurales y mineros, festivales de confraternización entre profesores y obreros, colonias escolares, escolares, etc...[ ...] No sólo se intenta dar una respuesta diferente a la cuestión social, sino elevar el nivel interno de la Universidad; formar hombres antes que sabios" ()Cantero, C. (2010).

Aunque no es una definición acabada, en ella se tienen en cuenta varios elementos importantes, entre ellos la precisión del doble papel de la extensión, en donde se beneficia tanto la sociedad y como la universidad.

La Reforma de Córdoba en 1918, en Argentina, origina una ruptura con las viejas imposiciones de la universidad en Latinoamérica, con su grado de vinculación al pueblo, el viejo anhelo de próceres anteriores, planteando el fortalecimiento de la función social de la Universidad a través de la Extensión Universitaria.

Proyectar la labor universitaria en el seno de la comunidad originó una nueva función como regularidad para la universidad: la función social, que significa en "... poner el saber universitario al servicio de la sociedad y hacer de sus problemas un aspecto importante de su ocupación" ().González, J. (1922).

En 1957 en la Primera Conferencia Latinoamericana de Extensión Universitaria y Difusión Cultural, la UNESCO emite una definición, que por su esencia, se puede clasificar como tradicional, dado que el vínculo se lleva a cabo teniendo en cuenta a la universidad como fuente de conocimientos para contribuir a la sociedad:

"Por su naturaleza, la extensión universitaria es misión y función orientadora de la universidad contemporánea, entendida como ejercicio de la vocación universitaria. Por su contenido y 
procedimiento, la extensión universitaria se funda en el conjunto de estudios y actividades filosóficas, científicas, artísticas y técnicas, mediante el cual se auscultan, exploran y recogen del medio social, nacional y universal, los problemas, datos y valores culturales que existen en todos los grupos sociales. Por sus finalidades, la extensión universitaria debe proponerse, como fines fundamentales proyectar dinámica y coordinadamente la cultura y vincular a todo el pueblo con la universidad" ().UDUAL. (1957).

Como se puede observar, la universidad observa a la sociedad y decide el vínculo a través de su potencial académico.

Pablo Freire en 1972, centrándose en la interacción universidad-sociedad, define la extensión a partir del dialogo que se debe dar entre ambos polos definiendo quien da y quien recibe.

"Un proceso educativo unidireccional, donde por un lado están los que dan, y por otro lado los que reciben. De ello es que defiende su concepción de comunicación horizontal y dialógica, con énfasis en una educación de aprendizaje compartido" (12).

En 1996 Gil González, en su tesis de doctorado, ofrece una definición con un carácter desarrollador:

"La Extensión Universitaria es el proceso universitario que, como parte del sistema de interacciones Universidad-Sociedad, tiene el propósito de promover el desarrollo cultural de la comunidad intra y extrauniversitaria, inmersa en los demás procesos principales de la educación superior: docencia (pregrado y postgrado) e investigación, con personalidad propia y aporta un producto final $o$ resultado de la labor universitaria. Como se ha dicho antes, es la función que expresa la cualidad del proceso. Por ello la relación dialéctica función proceso, que se expresa con carácter de ley, es la que permite desarrollar su gestión con eficiencia y eficacia" (13).

En la definición se precisa el desarrollo cultural tanto de la universidad como de la sociedad, aunque no se explicita cual es el elemento central para definir dicho desarrollo.

Por su parte Jesús Cruz (1998), define la extensión universitaria como:

"En otras palabras, la extensión universitaria adquiere su 'razón de ser' cuando vive y mira la institución desde fuera y desde allí, en su verdadero sitio, construye la interacción evaluadora de la investigación y la docencia. A partir de los 'saberes incorporados' de las culturas locales/regionales y a partir de los problemas de las comunidades, se demandan y se orientan los saberes académicos, buscando siempre que esos saberes se ordenen, necesariamente, en el juego interdisciplinario requerido por las comunidades" (8)

Aunque, se tratan los problemas comunitarios y se integran con los saberes académicos, no se especifica el carácter desarrollador y se retorna a una definición tradicionalista, como la que nos plantea CarlosTünnermann en el 2003:
"La extensión debe ser la mejor expresión de una integración creativa universidad-sociedad y su vínculo más idóneo; por lo que existe la posibilidad de hacer de esta función el eje de la acción universitaria, el hilo conductor de la inmersión social de la Universidad, con lo cual adquieren sus programas una extraordinaria relevancia en el quehacer de las instituciones de educación superior" (22).

Gonzalo Serna (2007), retoma la concepción desarrolladora de la extensión universitaria, reconociendo su papel transformador en la sociedad:

"Extensión universitaria, es la interacción entre la Universidad y los demás componentes del cuerpo social, a través de la cual ésta asume y cumple su compromiso de participación, en el proceso social de creación de la cultura y de liberación y transformación radical de la comunidad nacional" (21).

Sin embargo, años más tarde Gazzola (2011) retoma lo tradicionalista y se aparta de lo desarrollador:“....la pertinencia es la dimensión en que la extensión universitaria, desarrollándose a partir de la enseñanza y la investigación, concretiza la responsabilidad social de la universidad. Esta responsabilidad se expresa en la extensión de servicios y conocimientos a los segmentos de la sociedad que necesitan el saber producido en la universidad, en las diversas maneras con las cuales ese saber es traducido, entre otros, en acciones de atención a la salud, programas culturales, estrategias de innovación tecnológica, programas comunitarios de formación continua" (12)

Evidentemente en esta definición hay un sentido ofertista de la extensión universitaria, se trata de ver la universidad como una entidad de servicio, independiente de las necesidades sociales.

María Victoria González (2012) en su tesis de doctorado nos presenta una definición desarrolladora:

"Proceso formativo integrador y contextualizado, que de forma interdependiente jerarquiza los restantes procesos sustantivos hacia la preservación, desarrollo y socialización de la cultura local y universal, sobre la base de la diversidad cultural existente, lo que se concreta en la formación y promoción de una cultura general integral en el par universidad-sociedad, y contribuye al desarrollo cultural personal, comunitario y social" (15).

Como se puede comprender la definición anterior está centrada en el desarrollo cultural, que se debe alcanzar a través de los restantes procesos sustantivos y aunque se indica que contribuye al desarrollo cultural, no se indica que ese desarrollo debe ser la guía para extensión universitaria.

En el año 2013, Julio Cedeño en su tesis doctoral, formula una definición como sigue:

"Presencia e integración académica mediante la cual, la Universidad aporta a la sociedad en forma crítica y creadora, los resultados y logros de su docencia, su investigación, su cultura y sus servicios; y por medio de la cual, al conocer la realidad comunitaria y social, enriquece y redimensiona su 
currículo y estrategias de desarrollo de manera democrática, autónoma, crítica y creativa" (13).

No obstante se especifica un aporte creador, dándole un carácter desarrollador, la universidad aporta según sus procesos sustantivos, según su oferta. Esta definición es análoga a las definiciones que exponen la Universidad Nacional de la Plata de Argentina en su portal ()UNP y ().CANO, JA. (2014)

En las definiciones anteriores se han omitido algunos presupuestos importantes, Fernando Vecino en 1992 puntualizaba:

"Por definición la universidad es el centro cultural más importante en cualquier país. Dentro de su recinto se hace ciencia, se hace cultura, se nutre la mente y el espíritu. Por ello, la Universidad debe irradiar ciencia y cultura" ().Vecino, F. (1992).

En ese mismo Canteronos alerta:

"La universidad nació para la satisfacción de las necesidades sociales que implican acumular, trasmitir y desarrollar la cultura universal. No siempre las necesidades atendidas son las de la inmensa mayoría; por lo general la prioridad recae en las necesidades de la clase dominante, y cuando esta clase se ha olvidado de las necesidades de la mayoría, esta se lo recuerda a través de crisis, a través de revoluciones sociales. Ese ha sido el camino tortuoso y a la vez luminoso del desarrollo de la universidad" ().Cantero (2010)

De ambas sentencias se extrae una idea importante, que el centro de la extensión es la solución de las necesidades sociales y para ello la universidad no solo emplea sus recursos, también, recursos emanados de sus alianzas a través de la Interinstitucionalización y la internacionalización, lo que ratifica. Veras, $H$. (2010).

En la Segunda Conferencia Mundial París, julio de 2009, en su Comunicado Final proclamó, que, "se deben perseguir, al mismo tiempo, metas de equidad, pertinencia y calidad" (Tünnermann, 2010). La unión de equidad y pertinencia recuerda a los autores a una definición de desarrollo esclarecedora formulada por el PNUD, que adopta el concepto de desarrollo humano sostenible:

"Debemos unir el desarrollo sostenible y el desarrollo humano, y unirlos no sólo de palabra sino en los hechos, todos los días, en el terreno, en todo el mundo. El desarrollo humano sostenible es un desarrollo que no sólo genera crecimiento, sino que distribuye sus beneficios equitativamente; regenera el medio ambiente en vez de destruirlo; potencia a las personas en vez de marginarlas; amplía las opciones y oportunidades de las personas y les permite su participación en las decisiones que afectan sus vidas. El desarrollo humano sostenible es un desarrollo que está a favor de los pobres, a favor de la naturaleza, a favor del empleo y a favor de la mujer. Enfatiza el crecimiento, pero un crecimiento con empleos, un crecimiento con protección del medio ambiente, un crecimiento que potencia a la persona, un crecimiento con equidad" ().Del Toro, A. (2010).
Por lo que se toman como elementos esenciales, que debe tener el concepto de Extensión Universitaria en la actualidad:

1. Proceso integrador, sistémico y contextualizado.

2. soluciona los problemas de la comunidad intra y extrauniversitaria, democrática, autónoma, crítica y creativamente.

3. Con el fin de lograr el desarrollo humano sostenible

4. Fundamentalmente a través de la docencia y la investigación de la universidad y sus alianzas estratégicas.

En consecuencia, los autores formulan que la Extensión Universitaria es:

Un proceso sustantivo universitario integrador, sistémico y contextualizado, para la solución conjunta de los problemas de la sociedad y la universidad, de forma crítica, democrática, autónoma, y creativa a través, fundamentalmente, de los procesos de docencia e investigación de la universidad y de sus alianzas interinstitucionales e internacionales, con el fin de lograr el desarrollo humano sostenible.

Para comprender que ha sucedido en la formulación del concepto es necesario referirse a los modelos de extensión universitaria.

Explica (Serna (2007) que durante el siglo XX, los grandes cambios ocurridos en la educación superior han quedado evidenciados en cuatro modelos de extensión:

1. Altruista, que concibe la extensión como las acciones desinteresadas de los universitarios en favor de las poblaciones marginadas. Predominó en las primeras décadas del siglo XX.

2. Divulgativo, que pretende establecer las formas por las cuales pueden acercarse a la población los adelantos técnicos y las expresiones culturales originadas en la universidad. En este modelo la universidad se establece como centro de poder que no debe consultar a otros sectores vulnerables de la sociedad.

3. Concientizador, cuyo objetivo es la creación de conciencia, el estímulo de la capacidad de análisis crítico y la acción eficaz y transformadora. Este marco lo que conduce a una participación política activa.

4. Vinculatorio empresarial, el cual considera que la universidad debe tener como objetivo satisfacer las necesidades de las empresas. Este modelo toma fuerza a mitad de la década de los ochenta, y significó la aparición de beneficios económicos por sus servicios.

En la práctica estos modelos quedaron olvidados en el tiempo y a medida que se ha producido un aumento de la complejidad de la educación superior, se mezclan las características de uno y otro modelo, lo que hace imposible clasificar estos modelos con solo una palabra.(21)

Analizando las prácticas de las universidades latinoamericanas, González y González (2013), plantean tres modelos de extensión: 
1. Tradicional: que concibe la universidad como fuente de conocimiento y saberes, y establece una relación de saber institucionalizado con quien no lo posee. Este modelo es similar al altruista planteado por Serna en el 2007.

2. Economicista: que considera a la universidad como una empresa más que interactúa en el mercado, la cual adopta el rol de soporte científico y técnico del sector productivo, y donde el saber se organiza en función de la rentabilidad económica. Este modelo se asemeja al modelo vinculatorio empresarial planteado anteriormente.

3. Desarrollo integral: donde la universidad maneja el concepto de democratización del saber y asume la función social de contribuir a mejorar la calidad de vida de la sociedad. Este modelo apunta a la transformación social y económica de los pueblos ().González y González (2013)

Sin embargo, en las condiciones actuales, las universidades latinoamericanas han perdido la hegemonía total sobre el conocimiento, quizás producto a sus imperfecciones, entre ella la poca pertinencia social, por lo que las empresas han creado sus propios sistemas de desarrollo científico técnico, lo que les ha permitido compartir la hegemonía del conocimiento con la universidad, esta situación se puede observar en países en desarrollo, donde existen empresas con un nivel de conocimientos científico insertados en su tecnología, que no están en el contenido de los currículos universitario, lo que conduce a que el modelo economicista no sea significativo para estudiar profundamente al proceso de extensión.

En consecuencia de lo anterior prácticamente existen dos modelos generales (Tradicional y desarrollador), que se especifican en diferentes dimensiones. Así, aparecen; el modelo tradicional, que está basado en la búsqueda de una relación entre la universidad y la sociedad, teniendo en cuenta a la universidad como fuente de conocimientos para contribuir a la sociedad. La relación se consigue por diferentes vías:

Vinculatoria: relación simple entre la universidad y la sociedad.

Interdependiente: concibe un aprendizaje mutuo de la universidad y la sociedad

Integrativa: concibe una acción mutua de la universidad y la sociedad.

Desarrollador: como su nombre lo indica, tiene la misión de lograr el desarrollo y la dirección del mismo puede ser:

Cultural: Dirigida al desarrollo de la cultura universal.

Culturo céntrico: Dirigido a la promoción de la cultura universal.

Ofertista: Dirigido a la cultura que emana de los procesos de la universidad.

Participativo: La universidad participa en el desarrollo social.
Sustentable: Dirigido a la solución de los problemas sociales (que al ser creciente las necesidades sociales se hace sustentable).

\section{Conclusiones}

El concepto de extensión universitaria debe reflejar la necesaria vinculación universidad en las condiciones actuales de desarrollo, así como, reflejar que a través de ella, la universidad logra su pertinencia en alianza con otros centros e instituciones, pero debe, sobre todo reflejar que la universidad es el centro que irradia cultura a toda la sociedad.

Los modelos de extensión universitaria, a su vez, deben tener la base de su clasificación en la esencia de la universidad en su misión..

\section{Referencias}

1. Álvarez,C. La Universidad como Institución Social. Editorial Academia. La Habana, Cuba. 1996.

2. Arce, C.La cultura Universitaria basada en la Calidad y la http://www.csuca.edu.gt/Eventos/SIMPOSIUM/Ejes/ Cultura/ponenciahaciaunaculturauniv.pdf. 2005.

3. Barrios,O. Para Una definición del Trabajo Comunitario desde la Extensión Universitaria., Universidad de Oriente, Santiago de Cuba. 2006.

4. Betto,F. La extensión universitaria. Conferencia especial. Congreso Internacional Universidad 2012. 2012.

5. Bustos,C. La extensión universitaria una estrategia pedagógica eficaz. Universidad del Zulia. Venezuela.2010.

6. Cedeño,J.Estrategia de gestión de la extensión universitaria para la vinculación universidadcomunidad. Tesis en opción al grado científico de Doctor en Ciencias Pedagógicas. Camagüey.2013.

7. Claret del Valle,N. La gestión de la cultura extensionista desde la IES. Tesis en opción al grado científico de doctora en Ciencias Pedagógicas. Universidad de Oriente. Santiago de Cuba. 2009.

8. Cruz,J.La Extensión Universitaria: $\mathrm{Su}$ rol protagónico en los próximos quinquenios. España. UNA Documental.1998. No 12 (1-2).

9. Del Huerto,M.Propuesta de proyección estratégica para la extensión universitaria en la Facultad de Industrial-Economía de la UMCC. Tesis presentada en opción al título académico de Master en Desarrollo Cultural. Instituto Superior de Arte. Cuba. 2000.

La Extensión Universitaria como

vía para fortalecer los vínculos Universidad-Sociedad desde la promoción de salud Rev. Cubana de Salud Pública, (2007). 33(2).

10. Doria,M. Extensión universitaria frente a las necesidades sociales. Universidad del Zulia. Venezuela.2009. 
11. Freire,P. ¿Extensión o Comunicación? La Concientización en el Medio Rural. Argentina. 1972.

12. Gazzola,L. Análisis de la afición a la lectura en usuarios adolescentes de la red social Tuenti. Revista Interuniversitaria de Formación del Profesorado. Número 72 (25,3). Zaragoza.2011.

13. González,G. Un modelo de extensión universitaria para la Educación Superior cubana. Su aplicación en la Cultura Física y el Deporte. Tesis en opción al grado de Doctor en Ciencias Pedagógicas. Universidad de La Habana, La Habana 1996.

14. González-Moreno ,M. Formulación teóricometodológica de la promoción cultural de la investigación para la integración de los procesos universitarios extensión e investigación en el Instituto Superior Politécnico José Antonio Echeverría. Tesis en opción al grado científico de doctora en Ciencias de la Educación. Instituto Superior Politécnico José Antonio Echeverría Ciudad de la Habana, Cuba. 2006. 15. González,MV.Gestión de la extensión universitaria con enfoque intercultural en condiciones de universalización. Tesis en opción al grado científico de Doctor en Ciencias Pedagógicas. Camagüey.2012.

La extensión universitaria desde la perspectiva de la Educación Popular en el contexto de la nueva universidad cubana. VI Encuentro Internacional "Presencia de Paulo Freire". ISBN 978959-257-232-4. Universidad de Cienfuegos. 2010.

Extensión Universitaria en la universalización de la Educación Superior: una mirada desde la pedagogía intercultural. Revista electrónica. Humanidades Médicas. Vol. 11 No.2 mayoagosto/2011. Versión on-line. ISSN 1727-8120. Págs. 274-289 http:/www.humanidadesmedicas.sld.cu 2011

16. Hart,A. La extensión universitaria y la integración cultural. La extensión universitaria y la integración cultural. 1996.

17. Hernández,S. La dinámica de la extensión universitaria para favorecer la formación inicial del profesional de la educación en la escuela Secundaria Básica como micro universidad. Tesis presentada en opción al grado científico de Doctor en Ciencias Pedagógicas. Universidad de Holguín Oscar Lucero. 2011.

18. Horruitiner,P. La universidad cubana: el modelo de formación. Editorial Félix Varela 2008.

19. Reyes,M. La cultura científica desde los círculos de interés de pioneros en Secundaria Básica. Tesis en opción al grado científico de Doctor en Ciencias Pedagógicas. ISP "Félix Varela". Villa Clara. Cuba. 2007.

20. Sánchez,M. La Extensión ante los nuevos escenarios de la Universidad cubana: una visión. Tesis presentada en opción al título académico de Master en Desarrollo Cultural Comunitario., Universidad de Oriente, Santiago de Cuba.Cuba. 2003.

21. Serna,G.Misión social y modelos de extensión universitaria: del entusiasmo al desdén. En: Revista Iberoamericana de Educación, 43: 3-25. 2007.
22. Tünnermann,C. El nuevo concepto de extensión universitaria. En Memorias V Congreso Iberoamericano de extensión, México. 1998.

La universidad latinoamericana ante los retos del siglo XXI. Colección UDUAL.2003. P 69.

23. Vilá,D, et al. Paradigma Cultural. Sujeto y Extensión Universitaria de la Fragmentación a la Integración. Congreso Internacional. Universidad 2006.2006.

24. Zulueta,E. Visión y misión de la extensión universitaria frente a la dinámica transculturizadora de la globalización. 2010.

Bibliografías

Cano,J.Extensión universitaria y alternativas pedagógicas en la Universidad Latinoamericana. En La construcción del futuro: los retos de las Ciencias Sociales en México. Memorias del $4^{\circ}$ Congreso Nacional de Ciencias Sociales. COMECSU. México.2014.

Cantero,C. El Concepto de la Extensión Universitaria a lo largo de la historia. Revista Atalaya. Andalucía. España.2010.

Del Toro,A. La formación bioética del veterinario. Tesis en opción al grado de Doctor en Ciencias Pedagógicas. Camagüey. Cuba.2010.

Ferrer,T. "Extensión Universitaria y universidades populares en la España de entresiglos: una estrategia educativa de reforma social". Revista de Educación. N. extraordinario, pp. 95-113.1997.

González,M,y González,G. Extensión universitaria, proyección social o tercera misión?, una reflexión necesaria. Revista Congreso Universidad. Vol. II, No. 2.2013 .

González,J. La Revolución Universitaria, Buenos Aires, Argentina.1922.

Jiménez-Landi,A,.Breve historia de la Institución Libre de Enseñanza (1896-1939). Tébar. 2010

Mariás,J. Historia de la Filosofía - Manuales de la Revista Occidente. 9a. Edición, Madrid, España, pág. 11-23.1957.

Sastre,P; Zubiría,A y D’Andrea,R.Concepciones sobre extensión universitaria. Universidad Nacional del Centro de la Provincia de Buenos Aires. Argentina. 2012.

.Nuevas Perspectivas de la Pertinencia y Calidad de la Educación Superior. Boletín IESALC Informa. UNESCO.2010.

UDUAL. Primera Conferencia Latinoamericana de Extensión Universitaria y Difusión Cultural. . (1957).

UNP. Portal de la vicerrectoría de Extensión. http://www.unlp.edu.ar/articulo/2008/4/10/extension_ apertura_institucional 2014.

Vecino,F. Intervención en el Seminario de Dirigentes de la Educación Superior- MES. Habana.1992.

Veras,H. Internacionalización y Cooperación universitaria. II Encuentro Internacional de Rectores de UNIVERSIA. Ponencias. Guadalajara. México.2010. 